\title{
Exploring electronic coupling of optical and phonon excitations at the nanoscale
}

Juan Carlos Idrobo ${ }^{1}$, Andrea Konečná ${ }^{2}$, Kate Reidy ${ }^{3}$, Eugene Park ${ }^{4}$, Pavel Gallina ${ }^{5}$, Tomáš Šikola ${ }^{6}$, Frances Ross ${ }^{7}$ and F. Javier Garcia de Abajo ${ }^{8}$

${ }^{1}$ Oak Ridge National Laboratory, Oak Ridge, Tennessee, United States, ${ }^{2}$ ICFO-Institut de Ciencies Fotoniques, United States, ${ }^{3}$ Massachusetts Institute of Technology (MIT), United States, ${ }^{4}$ Massachusetts Institute of Technology, Massachusetts, United States, ${ }^{5}$ CEITEC, BUT, Brno, Moravskoslezsky kraj, Czech Republic, ${ }^{6}$ Brno University of Technology, United States, ${ }^{7}$ MIT, United States, ${ }^{8}$ ICFO-Institut de Ciencies Fotoniques, Castelldefels, Catalonia, Spain

A new domain for electron energy-loss spectroscopy (EELS) is now available with the recent development of monochromators in scanning transmission electron microscopy (STEM) [1]. This has allowed to study the nature of low energy $(<1 \mathrm{eV})$ excitations in materials with an unprecedented combination of spectral and spatial resolution. Two recent examples are the detection of vibrations of different carbon isotopes in amino acids [2] and the direct atomic-scale observation of how a single atomic impurity can influence the phonon response of a material [3].

Another area in which EELS can now contribute is in the understanding of mutual coupling between quasiparticles such as phonons, plasmons, or excitons. For instance, it has been recently shown that it is possible to detect, and spatially map, a strong phonon-plasmon coupling between a silver nanowire and a thin substrate of hexagonal boron nitride [4].

Similarly, recent work by Yankovich and colleagues [5] presented direct evidence of plexcitons (plasmonexciton polaritons) in a silver nano-triangle supported on a few-layer sheet of WS2. However, the study reported that the plexcitons had anomalies (different intensity strengths) that could not be directly explained by the experiments. Shape irregularities of the nano-triangle as well as a moiré potential, arising from the mismatch on the interface between $\mathrm{Ag}$ and $\mathrm{WS}_{2}$, were suggested as possible culprits.

Here, we will present a methodology of how the spatial dependence of an exciton-plasmon and phononplasmon coupling can be easily determined from EELS data guided by theoretical calculations. We will also show our experimental efforts to locally study electronic states associated with moiré potentials at a 2D/3D interface between few-layer $\mathrm{MoS}_{2}$ and an Au nanostructure [6], as shown in Figure 1. Finally, we will discuss the future experimental conditions required to study electronic excitations such as those arising from superconducting and topological materials [7, 8]. 


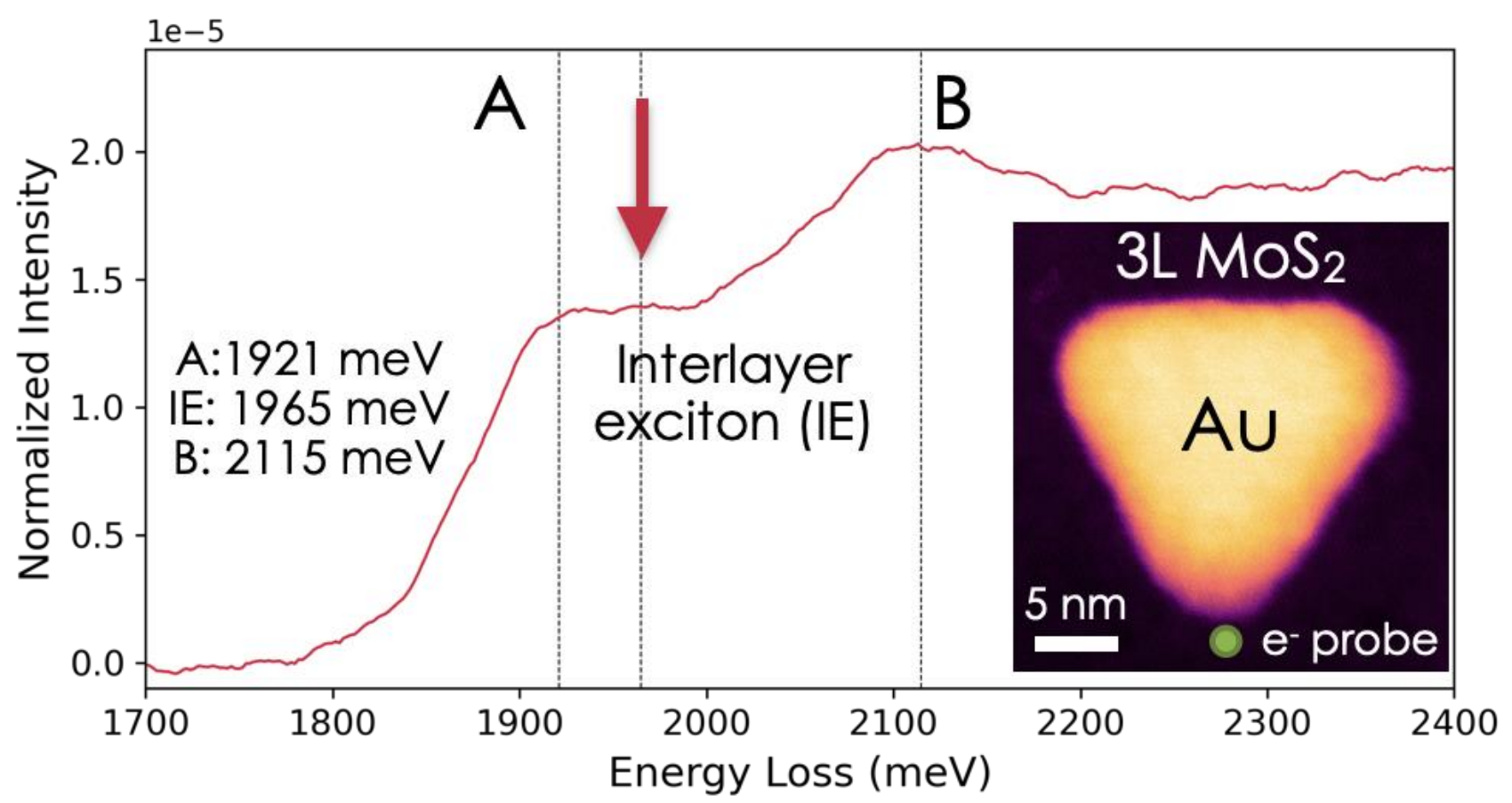

Figure 1. Averaged EEL spectrum of a Au nanostructure on top of 3-layer $\mathrm{MoS}_{2 \text { acquired at room temperature with an energy }}$ resolution of $20 \mathrm{meV}$, using a Nion monochromated aberration-corrected (MAC)-STEM operated at $60 \mathrm{kV}$. The inset shows a Z-contrast image of the system studied and the electron probe location where the spectrum was acquired

References

[1] O. L. Krivanek, et al., Phil. Trans. R. Soc. A 367 (2009), p. 3683.

[2] J.A Hachtel, et al., Science 363 (2019), p. 525.

[3] F.S. Hage, et al., Science 367 (2020), p. 1124.

[4] L. Tizei, et al., Nano Lett. 20, (2020), p. 2973.

[5] A.B. Yankovich, et al., Nano Lett. 19, (2019), p. 8171.

[6] K. Reidy, et al., Nature Comm. (2021), doi:10.1038/s41467-021-21363-5.

[7] J.C. Idrobo, Nature Rev. Mat. 6, (2021), p. 100.

[8] The EELS measurements were supported by the Center for Nanophase Materials Sciences, which is a Department of Energy Office of Science User Facility (J.C.I.). This work also made use of facilities and instrumentation supported by NSF through the Massachusetts Institute of Technology Materials Research Science and Engineering Center DMR-1419807, as well as facilities at MIT.nano. K.R. acknowledges funding from a MIT MathWorks Engineering Fellowship and an OGE MIT Fellowship. This research was conducted, in part, using instrumentation within ORNL's Materials Characterization Core provided by UT-Battelle, LLC under Contract No. DE-AC05-00OR22725 with the U.S. Department of Energy. Theoretical calculations used resources of the National Energy Research Scientific Computing Center, which is supported by the Office of Science of the U.S. Department of Energy under Contract No. DEAC02-05CH11231. This work has been also supported in part by the European Research Council (Advanced Grant 789104-eNANO), the European Commission (Horizon 2020 Grants 101017720 FETProactive EBEAM, 964591-SMART-electron, and 810626 - SINNCE), the Czech GAČR 20-28573S, the Spanish MINECO (MAT2017-88492-R and Severo Ochoa CEX2019-000910-S), the Catalan CERCA Program, and Fundaciós Cellex and Mir-Puig. 